\title{
AN INFINITE FAMILY OF GROMOLL-MEYER SPHERES
}

\author{
CARLOS DURÁN, THOMAS PÜTTMANN, AND A. RIGAS
}

\begin{abstract}
We construct a new infinite family of models of exotic 7-spheres. These models are direct generalizations of the Gromoll-Meyer sphere. From their symmetries, geodesics and submanifolds half of them are closer to the standard 7-sphere than any other known model for an exotic 7-sphere.
\end{abstract}

\section{INTRODUCTION}

This paper provides a new geometric way to construct all exotic 7-spheres. Exotic spheres are differentiable manifolds that are homeomorphic but not diffeomorphic to standard spheres. The first examples were found by Milnor Mi1 in 1956 among the $\mathbb{S}^{3}$-bundles over $\mathbb{S}^{4}$. It turned out that 7 is the smallest dimension where exotic spheres can occur except possibly in the special dimension 4 . In any dimension $n>4$ the exotic spheres and the standard sphere form a finite abelian group: the group $\Theta_{n}$ of (orientation preserving diffeomorphism classes of) homotopy spheres $\left[\mathrm{KM}\right.$. The inverse element in $\Theta_{n}$ can be obtained by a change of orientation. In dimension 7 we have $\Theta_{7} \approx \mathbb{Z}_{28}$. Hence, ignoring orientation there are 14 exotic 7 -spheres. From these 14 exotic 7 -spheres four (corresponding to $\left.2,5,9,12,16,19,23,26 \in \mathbb{Z}_{28}\right)$ are not diffeomorphic to an $\mathbb{S}^{3}$-bundle over $\mathbb{S}^{4}$ EK.

In 1974 Gromoll and Meyer [GM] constructed an exotic 7 -sphere, $\Sigma_{\mathrm{GM}}^{7}$, as quotient of the compact group $\operatorname{Sp}(2)$ by a two-sided $\mathbb{S}^{3}$-action. This construction provided $\Sigma_{\mathrm{GM}}^{7}$ automatically with a metric of nonnegative sectional curvature $(K \geq 0)$. The Gromoll-Meyer sphere $\Sigma_{\mathrm{GM}}^{7}$ was the only exotic sphere known to admit such a metric until 1999 when Grove and Ziller [GZ] constructed metrics with $K \geq 0$ on all Milnor spheres, i.e., on all exotic 7 -spheres that are $\mathbb{S}^{3}$-bundles over $\mathbb{S}^{4}$. In 2002 Totaro [To and independently Kapovitch and Ziller [KZ] showed that $\Sigma_{\mathrm{GM}}^{7}$ is the only exotic sphere that can be modeled by a biquotient of a compact group and thus underlined the singular status of the Gromoll-Meyer sphere among all models for exotic spheres.

We nevertheless provide an elementary and direct generalization of the GromollMeyer construction. The essential components in this construction are natural selfmaps of $\mathbb{S}^{7}$, namely, the $n$-powers of unit octonions, $n \in \mathbb{Z}$. In terms of quaternions these maps are defined by

$$
\rho_{n}: \mathbb{S}^{7} \rightarrow \mathbb{S}^{7}, \quad\left(\begin{array}{c}
\cos t+p \sin t \\
w \sin t
\end{array}\right) \mapsto\left(\begin{array}{c}
\cos n t+p \sin n t \\
w \sin n t
\end{array}\right)
$$

C. Duran and A. Rigas were supported by CNPq. C. Duran was also supported by FAPESP grant 03/016789 and FAEPEX grant 15406. T. Püttmann was supported by a DFG Heisenberg fellowship and by the DFG priority program SPP 1154 "Globale Differentialgeometrie". 
where $p \in \operatorname{Im} \mathbb{H}$ and $w \in \mathbb{H}$ with $|p|^{2}+|w|^{2}=1$. Let $\langle\langle u, v\rangle\rangle:=\bar{u}^{\mathrm{t}} v$ denote the standard Hermitian product on $\mathbb{H}^{2}$. The submanifolds

$$
E_{n}^{10}:=\left\{(u, v) \in \mathbb{S}^{7} \times \mathbb{S}^{7} \mid\left\langle\left\langle\rho_{n}(u), v\right\rangle\right\rangle=0\right\}
$$

come equipped with a free action of the unit quaternions:

$$
\mathbb{S}^{3} \times E_{n}^{10} \rightarrow E_{n}^{10}, \quad q \star(u, v)=(q u \bar{q}, q v) .
$$

Here, $q u \bar{q}$ means that the two quaternionic components of $u$ are simultaneously conjugated by $q \in \mathbb{S}^{3}$. The quotient of $E_{n}^{10}$ by the free $\star$-action is a smooth manifold

$$
\Sigma_{n}^{7}:=E_{n}^{10} / \mathbb{S}^{3} .
$$

For $n=1$ we have $E_{1}^{10}=\operatorname{Sp}(2)$ (the group of quaternionic $2 \times 2$ matrices $A$ with $\left.\bar{A}^{\mathrm{t}} A=11\right)$ and the $\star$-action is the original Gromoll-Meyer action. Hence, $\Sigma_{1}^{7}=\Sigma_{\mathrm{GM}}^{7}$. It is also easy to see that $\Sigma_{0}^{7}$ is diffeomorphic to $\mathbb{S}^{7}$.

Theorem 1. The differentiable manifold $\Sigma_{n}^{7}$ is a homotopy sphere and represents the $(n \bmod 28)$-th element in $\Theta_{7} \approx \mathbb{Z}_{28}$.

Let $\mathbb{Z}_{2} \times \mathbb{Z}_{2}$ denote the diagonal matrices of $\mathrm{O}(2) \subset \mathrm{Sp}(2)$. All $E_{n}^{10}$ admit a smooth action of $\mathbb{Z}_{2} \times \mathbb{Z}_{2} \times \mathbb{S}^{3}$ that commutes with the free $\star$-action:

$$
\begin{gathered}
\mathbb{Z}_{2} \times \mathbb{Z}_{2} \times E_{n}^{10} \rightarrow E_{n}^{10}, \quad B \bullet(u, v)=(B u, B v), \\
\mathbb{S}^{3} \times E_{n}^{10} \rightarrow E_{n}^{10}, \quad q \bullet(u, v)=(u, v \bar{q}) .
\end{gathered}
$$

The induced effective action on $\Sigma_{n}^{7}$ is an action of $\mathbb{Z}_{2} \times \mathbb{Z}_{2} \times \mathrm{SO}(3)$ where $\mathrm{SO}(3)=$ $\mathbb{S}^{3} /\{ \pm 1\}$. On $\Sigma_{0}^{7}$ this action can be identified with the linear action

$$
(B, \pm q) \cdot(x, u)=(B x, B q u \bar{q})
$$

on $\mathbb{S}^{7} \subset \mathbb{R}^{2} \times(\operatorname{Im} \mathbb{H})^{2}$. On $\Sigma_{1}^{7}=\Sigma_{\mathrm{GM}}^{7}$ the action coincides with the subaction of the $\mathrm{O}(2) \times \mathrm{SO}(3)$-action given in $\mathrm{GM}$.

The surprising fact is the following even/odd grading of the $\Sigma_{n}^{7}$ :

Theorem 2. All $\Sigma_{n}^{7}$ with even $n$ are equivariantly homeomorphic to $\mathbb{S}^{7}$ with the linear $\mathbb{Z}_{2} \times \mathbb{Z}_{2} \times \mathrm{SO}(3)$-action given above. All $\Sigma_{n}^{7}$ with odd $n$ are equivariantly homeomorphic to the Gromoll-Meyer sphere $\Sigma_{\mathrm{GM}}^{7}$ with the above $\mathbb{Z}_{2} \times \mathbb{Z}_{2} \times \mathrm{SO}(3)$ action. If $n$ is even all fixed point sets in $\Sigma_{n}^{7}$ are spheres while if $n$ is odd there are also 3-dimensional fixed point sets with fundamental groups $\mathbb{Z}_{2}$ and $\mathbb{Z}_{3}$.

The even/odd grading of the $\Sigma_{n}^{7}$ also transfers to some of the invariant submanifolds. The most important one is $\Sigma_{n}^{5}$ whose preimage under the map $E_{n}^{10} \rightarrow \Sigma_{n}^{7}$ consists of points $(u, v)$ where both quaternionic components of $u$ are purely imaginary.

Proposition 3. $\Sigma_{n}^{5}$ is $\mathbb{Z}_{2} \times \mathbb{Z}_{2} \times \mathrm{SO}(3)$-equivariantly diffeomorphic to $\mathbb{S}^{5} \subset(\operatorname{Im} \mathbb{H})^{2}$ with the linear action $(B, \pm q) \cdot u=B q u \bar{q}$ if $n$ is even and to the Brieskorn sphere $W_{3}^{5}$ if $n$ is odd. The subsphere $\Sigma_{n}^{5}$ is minimal for every $\{ \pm \mathbb{1}\} \times \mathrm{SO}(3)$-invariant metric on $\Sigma_{n}^{7}$. 
Recall here that the Brieskorn sphere $W_{d}^{5}$ with $d \in \mathbb{N}$ is the intersection of the unit sphere in $\mathbb{C}^{4}=\mathbb{C} \oplus \mathbb{C}^{3}$ with the complex hypersurface

$$
z_{0}^{3}+z_{1}^{2}+z_{2}^{2}+z_{3}^{2}=0
$$

and that there is a natural $\mathrm{O}(2) \times \mathrm{SO}(3)$-action on $W_{d}^{5}$ :

$$
\begin{aligned}
& \mathrm{O}(2) \times \mathrm{SO}(3) \times W_{d}^{5} \rightarrow W_{d}^{5}, \\
& \left(\left(\begin{array}{c}
\cos \theta-\sin \theta \\
\sin \theta
\end{array}-\cos \theta\right), A\right) \cdot\left(z_{0}, z\right)=\left(e^{2 i \theta} z_{0}, e^{d i \theta} A z\right), \\
& \left(\left(\begin{array}{rr}
1 & 0 \\
0 & -1
\end{array}\right), A\right) \cdot\left(z_{0}, z\right)=\left(\bar{z}_{0}, A \bar{z}\right) .
\end{aligned}
$$

The classification theorems of Jänich and Hsiang-Hsiang imply that for $G=\mathrm{O}(2) \times$ $\mathrm{SO}(3)$ and even for the smaller group $G=\{ \pm \mathbb{1}\} \times \mathrm{SO}(3)$ the Brieskorn sphere $W_{d}^{5}$ is not $G$-equivariantly homeomorphic to $\mathbb{S}^{5}$ with any linear action, see HMa. However, $W_{d}^{5}$ is $\mathrm{SO}(3)$-equivariantly diffeomorphic to $\mathbb{S}^{5}$. In the case $d=3$ an explicit formula for such a diffeomorphism is given in $\mathrm{DP}$.

The invariant subsphere $\Sigma_{n}^{5}$ is dual to the invariant circle $\Sigma_{n}^{1}$ whose preimage under the map $E_{n}^{10} \rightarrow \Sigma_{n}^{7}$ consists of points $(u, v)$ for which both components of $u$ are real. These two dual submanifolds play a central role for the geodesic geometry of $\Sigma_{n}^{7}$. We construct a one parameter family of $\mathbb{Z}_{2} \times \mathbb{Z}_{2} \times \mathrm{SO}(3)$-invariant metrics $\langle\cdot, \cdot\rangle_{\nu}$ on each $\Sigma_{n}^{7}$ with the following property:

Theorem 4. All points $p \in \Sigma_{n}^{1}$ have the wiedersehen property, i.e., every unit speed geodesic $\gamma$ in $\Sigma_{n}^{7}$ with $\gamma(0)=p$ is length minimizing on $[0, \pi[$ and obeys $\gamma(\pi)=-p$ and $\gamma(2 \pi)=p$. Moreover, $\Sigma_{n}^{1}$ and $\Sigma_{n}^{5}$ have constant distance $\frac{\pi}{2}$ and the map $\Sigma_{n}^{1} * \Sigma_{n}^{5} \rightarrow \Sigma_{n}^{7}$ that maps $(x, y, t)$ to $\gamma(t)$, where $\gamma:\left[0, \frac{\pi}{2}\right] \rightarrow \Sigma^{7}$ is the unique unit speed geodesic segment from $x$ to $y$, is a homeomorphism.

This invariant geodesic join structure actually is the key to prove Theorem 1 and Theorem 2. In the particular case of exotic 7-spheres our method is an improvement over the general construction that equips all exotic spheres with pointed wiedersehen metrics $\mathrm{Bs}$.

The even/odd grading of the $\Sigma_{n}^{7}$ is in contrast to what happens for the Milnor spheres $M_{k, l}^{7}$ and the Brieskorn spheres $W_{6 n-1,3}^{7}$.

The Milnor sphere $M_{k, l}^{7}$ with $k+l=1$ is defined by gluing two copies of $\mathbb{H} \times \mathbb{S}^{3}$ along $(\mathbb{H} \backslash\{0\}) \times \mathbb{S}^{3}$ by the map

$$
(u, v) \mapsto\left(\frac{u}{|u|^{2}},\left(\frac{u}{|u|}\right)^{k} v\left(\frac{u}{|u|}\right)^{l}\right) .
$$

For convenience, we set $M_{k, l}^{7}=M_{d}^{7}$ where $d=k-l$ is odd. The Milnor sphere $M_{d}^{7}$ represents the $\frac{d^{2}-1}{8}$-th element in $\Theta_{7}$, see [EK]. There is a natural $\mathrm{SO}(3)=$ $\mathbb{S}^{3} /\{ \pm 1\}$-action on $M_{d}^{7}$ which is in both charts defined by

$$
\pm q \bullet(u, v)=(q u \bar{q}, q v \bar{q}) .
$$

Davis Da has shown that $M_{d}^{7}$ is $\mathrm{SO}(3)$-equivariantly diffeomorphic to $M_{d^{\prime}}^{7}$ if and only if $d^{\prime}= \pm d$ and that all $M_{d}^{7}$ are $\mathrm{SO}(3)$-equivariantly homeomorphic to $\mathbb{S}^{7} \subset \mathbb{H}^{2}$ 
with the linear $\mathrm{SO}(3)$-action given by $( \pm q, u) \mapsto q u \bar{q}$. We show that the latter situation changes when one extends the $\mathrm{SO}(3)$-action by the commuting involution

$$
(u, v) \mapsto(u,-v)
$$

in both charts. This involution fixes all points in the base of the bundle $M_{d}^{7} \rightarrow \mathbb{S}^{4}$ and induces the antipodal map on all the $\mathbb{S}^{3}$-fibers. For consistency, the group generated by $\mathrm{SO}(3)$ and the involution is denoted by $\{ \pm \mathbb{1}\} \times \mathrm{SO}(3)$.

Theorem 5. The fixed point set of the involution $(-11, \pm i)$ on $M_{d}^{7}$ is a 3-dimensional lens space with fundamental group $\mathbb{Z}_{|d|}$. Hence, $M_{d}^{7}$ is $\{ \pm \mathbb{1}\} \times \mathrm{SO}(3)$-equivariantly homeomorphic to $M_{d^{\prime}}^{7}$ if and only if $d= \pm d^{\prime}$. Moreover, for $|d|>3$ none of the $M_{d}^{7}$ are $\{ \pm \mathbb{1}\} \times \mathrm{SO}(3)$-equivariantly homeomorphic to any of the $\Sigma_{n}^{7}$.

This theorem is a consequence of Theorem [5.1 which is the analogue of Proposition 3 for the Milnor spheres.

Grove and Ziller GZ constructed $\mathrm{SO}(3)$-actions on $M_{d}^{7}$ that are entirely different from the $\mathrm{SO}(3)$-actions on $M_{d}^{7}$ and $\Sigma_{n}^{7}$ above. The $\mathrm{SO}(3)$-actions on $M_{d}^{7}$ and $\Sigma_{n}^{7}$ fix a circle pointwise while the Grove-Ziller actions are almost free.

The Brieskorn sphere $W_{6 n-1,3}^{7}$ is defined by the intersection of the the unit sphere $\mathbb{S}^{9} \subset \mathbb{C}^{5}=\mathbb{C} \oplus \mathbb{C} \oplus \mathbb{C}^{3}$ with the complex hypersurface

$$
w^{6 n-1}+z_{0}^{3}+z_{1}^{2}+z_{2}^{2}+z_{3}^{2}=0 .
$$

It represents the $(n \bmod 28)$-th homotopy sphere in $\Theta_{7}$ (see $[\mathrm{Bk})$ and admits the natural $\mathrm{SO}(3)$-action $\left(A,\left(w, z_{0}, z\right)\right) \mapsto\left(w, z_{0}, A z\right)$.

Theorem 6. None of the $W_{6 n-1,3}^{7}$ are $\mathrm{SO}(3)$-equivariantly diffeomorphic to any of the $\Sigma_{n}^{7}$ or to any of the $M_{k, l}^{7}$.

In particular, $W_{6 n-1,3}^{7}$ is not $\mathrm{SO}(3)$-equivariantly homeomorphic to the join of a circle and $W_{3}^{5}$. Thus, the equivariant topology of the $\Sigma_{n}^{7}$ with odd $n$ is much more determined by the equivariant topology of $W_{3}^{5}$ than the equivariant topology of $W_{6 n-1,3}^{7}$ although the latter contain $W_{3}^{5}$ in a much more obvious way (just by setting $w=0)$.

Many of the constructions in this paper generalize the constructions given in [DP for the original Gromoll-Meyer sphere $\Sigma_{\mathrm{GM}}^{7}$.

The authors would like to thank Uwe Abresch for several useful discussions and Wolfgang Ziller for many valuable suggestions.

\section{A Construction of the $\mathbb{S}^{3}$-PRINCIPAL BUndLes OVER $\mathbb{S}^{7}$}

Recall from the introduction the definition of $E_{n}^{10} \subset \mathbb{S}^{7} \times \mathbb{S}^{7}$ :

$$
E_{n}^{10}=\left\{(u, v) \in \mathbb{S}^{7} \times \mathbb{S}^{7}\left|\left\langle\rho_{n}(u), v\right\rangle\right\rangle=0\right\} .
$$

For $n=1$ the space $E_{1}^{10}$ can be equivalently seen as the group $\operatorname{Sp}(2)$ of $2 \times 2$ quaternionic matrices $A$ such that $\bar{A}^{\mathrm{t}} A=\mathbb{1}$. The standard projection $\operatorname{Sp}(2) \rightarrow \mathbb{S}^{7}$, $A=(u, v) \mapsto u$ turns $\operatorname{Sp}(2)$ into an $\mathbb{S}^{3}$-principal bundle over $\mathbb{S}^{7}$. 
Lemma 2.1. $E_{n}^{10}$ is the pull-back of $\operatorname{Sp}(2)$ by the map $\rho_{n}: \mathbb{S}^{7} \rightarrow \mathbb{S}^{n}$.

Proof. By the usual explicit construction, the total space of the pull-back bundle $\rho_{n}^{*}(\operatorname{Sp}(2))$ is the submanifold of $\mathbb{S}^{7} \times \operatorname{Sp}(2)$ consisting of all pairs $(u, A)$ such that $\rho_{n}(u)$ is the first colum of $A$. It is evident, however, that in this construction we $\log$ the first column of $A$ twice. Eliminating this redundancy leads to the definition of $E_{n}^{10}$ above. This in particular shows that $E_{n}^{10}$ is a submanifold of $\mathbb{S}^{7} \times \mathbb{S}^{7}$.

Corollary 2.2. $E_{n}^{10}$ is an $\mathbb{S}^{3}$-principal bundle over $\mathbb{S}^{7}$ classified by $n \bmod 12$.

Proof. The $\mathbb{S}^{3}$-principal bundles over $\mathbb{S}^{7}$ are classified by $\pi_{6}\left(\mathbb{S}^{3}\right) \approx \mathbb{Z}_{12}$ and the characteristic map of the bundle $\operatorname{Sp}(2) \rightarrow \mathbb{S}^{7}$ generates $\pi_{6}\left(\mathbb{S}^{3}\right)$ (see $\underline{\mathrm{Hu}}$ or DMR for a more explicit reference). The map $\rho_{n}$ has degree $n$.

The principal bundle map $E_{n}^{10} \rightarrow \mathbb{S}^{7}$ is given by the projection to the first column. The corresponding free $\mathbb{S}^{3}$-action on $E_{n}^{10}$ is given by

$$
\mathbb{S}^{3} \times E_{n}^{10} \rightarrow E_{n}^{10}, \quad q \bullet(u, v)=(u, v \bar{q}) .
$$

The map $\tilde{\rho}_{n}$ in the pull-back diagram

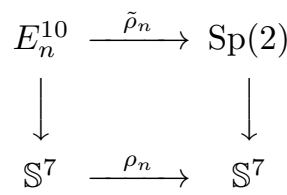

takes the explicit form

$$
\tilde{\rho}_{n}: E_{n}^{10} \rightarrow \operatorname{Sp}(2), \quad(u, v) \mapsto\left(\rho_{n}(u), v\right) .
$$

Recall from the introduction that there is a free $\mathbb{S}^{3}$-action $q \star(u, v)=(q u \bar{q}, q v)$ on $E_{n}^{10}$ that commutes with the $\bullet$-action and whose orbit space is the smooth manifold $\Sigma_{n}^{7}$. The pull-back diagram above extends to the following commutative diagram:

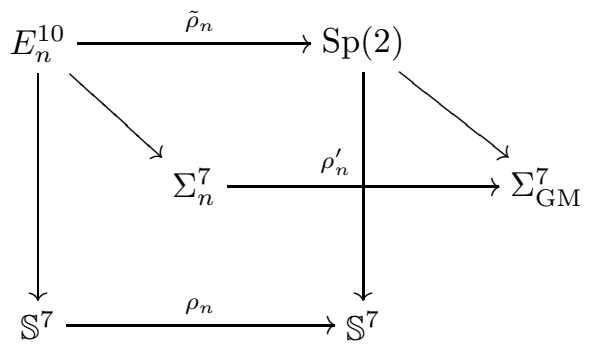

The degree of the induced map $\rho_{n}^{\prime}: \Sigma_{n}^{7} \rightarrow \Sigma_{\mathrm{GM}}^{7}$ is $n$. The proof that $\Sigma_{n}^{7}$ represents the $(n \bmod 28)$-th element of $\Theta_{7}$ requires several geometric constructions and is

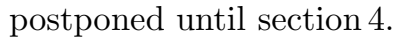

Each principal bundle $E_{n}^{10}$ admits a natural action of $\mathbb{Z}_{2} \times \mathbb{Z}_{2} \times \mathbb{S}^{3} \times \mathbb{S}^{3} \times \mathbb{S}^{3}$, where $\mathbb{Z}_{2} \times \mathbb{Z}_{2}$ denotes the diagonal matrices in $\mathrm{O}(2) \subset \mathrm{Sp}(2)$ :

$$
\begin{aligned}
& \mathbb{Z}_{2} \times \mathbb{Z}_{2} \times E_{n}^{10} \rightarrow E_{n}^{10}, \quad B \cdot(u, v)=(B u, B v), \\
& \mathbb{S}^{3} \times \mathbb{S}^{3} \times \mathbb{S}^{3} \times E_{n}^{10} \rightarrow E_{n}^{10}, \quad\left(q_{1}, q_{2}, q_{3}\right) \cdot\left(\begin{array}{lll}
u_{1} & v_{1} \\
u_{2} & v_{2}
\end{array}\right)=\left(\begin{array}{lll}
q_{1} u_{1} \bar{q}_{1} & q_{1} v_{1} \bar{q}_{3} \\
q_{2} u_{2} \bar{q}_{1} & q_{2} v_{2} \bar{q}_{3}
\end{array}\right) .
\end{aligned}
$$

Lemma 2.3. This action on $E_{n}^{10}$ is of cohomogeneity 2. 
Proof. The third $\mathbb{S}^{3}$-factor yields the principal action related to the bundle $E_{n}^{10} \rightarrow$ $\mathbb{S}^{7},(u, v) \mapsto u$, i.e., this $\mathbb{S}^{3}$-factor acts simply transitively on the fiber over any $u \in \mathbb{S}^{7}$. The action of the first two $\mathbb{S}^{3}$-factors on $\mathbb{S}^{7}$ has kernel $\{ \pm(1,1)\}$ and induces a standard linear $\mathrm{SO}(4)$-action on $\mathbb{S}^{7}$. By applying all three $\mathbb{S}^{3}$-factors one can transform an arbitrary point in $E_{n}^{10}$ to a point of the form

$$
\left(\begin{array}{cc}
\cos t+i \cos s \sin t & -\sin s \sin n t \\
\sin s \sin t & \cos n t-i \cos s \sin n t
\end{array}\right) .
$$

The diagonal in the first two $\mathbb{S}^{3}$-factors gives the Gromoll-Meyer action $\star$ corresponding to the principal bundle $E_{n}^{10} \rightarrow \Sigma_{n}^{7}$. The third $\mathbb{S}^{3}$-factor and the $\mathbb{Z}_{2} \times \mathbb{Z}_{2^{-}}$ factor yield the effective $\mathbb{Z}_{2} \times \mathbb{Z}_{2} \times \mathrm{SO}(3)$-action $\bullet$ on $\Sigma_{n}^{7}$ from the introduction. It is an interesting question for which $n$ this $\bullet$-action can be extended. The maximum dimension of any compact differentiable transformation group of an exotic 7-sphere is four $\mathrm{St}$. On the original Gromoll-Meyer sphere $\Sigma_{\mathrm{GM}}^{7}=\Sigma_{1}^{7}$ there is a natural $\mathrm{O}(2) \times \mathrm{SO}(3)$-action. It is induced by the action

$$
\mathrm{O}(2) \times \mathrm{SO}(3) \times \mathrm{Sp}(2) \rightarrow \mathrm{Sp}(2), \quad(A, q) \bullet(u, v) \mapsto(A u, A v \bar{q})
$$

on $\operatorname{Sp}(2)=E_{1}^{10}$ and extends the $\bullet$-action naturally. A corresponding $\mathrm{O}(2) \times \mathrm{SO}(3)$ action exists of course on $\Sigma_{-1}^{7}$. On $\Sigma_{0}^{7}$ an $\mathrm{O}(2) \times \mathrm{SO}(3)$-action is induced by the action

$$
\mathrm{O}(2) \times \mathrm{SO}(3) \times E_{0}^{10} \rightarrow E_{0}^{10}, \quad(A, q) \bullet(u, v) \mapsto(A u, v \bar{q}) .
$$

On the other $\Sigma_{n}^{7}$ with $n \neq-1,0,1$, however, it seems likely that the $\mathbb{Z}_{2} \times \mathbb{Z}_{2} \times \mathrm{SO}(3)$ action cannot be extended to any larger group, see Remark 4.5

Question 2.4. Which $E_{n}^{10}$ admit Riemannian metrics with $K \geq 0$ that are invariant under the cohomogeneity 2 action above? If some $E_{n}^{10}$ admits such a metric then by the O'Neill formulas the induced metric on $\Sigma_{n}^{7}$ also has $K \geq 0$. This would be particularly interesting for those $\Sigma_{n}^{7}$ that are not diffeomorphic to $\mathbb{S}^{3}$-bundles over $\mathbb{S}^{4}$ since on such exotic spheres no metrics with $K \geq 0$ are known so far.

Remark 2.5. While there are twelve $\mathbb{S}^{3}$-principal bundles over $\mathbb{S}^{7}$ there are 28 homotopy 7 -spheres. This means in particular that some $\Sigma_{n}^{7}$ are quotients of trivial bundles $E_{n}^{10}$. This phenomenon is well-known from surgery theory (see Wa inexplicit way.

Remark 2.6. Grove and Ziller GZ constructed cohomogeneity one metrics with $K \geq 0$ on all $\mathbb{S}^{3} \times \mathbb{S}^{3}$-principal bundles over $\mathbb{S}^{4}$. It is known that the $(n \bmod 12)$-th $\mathbb{S}^{3}$-principal bundle over $\mathbb{S}^{4}$ is diffeomorphic to an $\mathbb{S}^{3} \times \mathbb{S}^{3}$-principal bundles over $\mathbb{S}^{4}$ if and only if $n \bmod 12 \in\{0,1,3,4,6,7,9,10\}$. It is easy to see that the set of all integers $n$ with $n \bmod 12 \in\{0,1,3,4,6,7,9,10\}$ maps surjectively on $\mathbb{Z}_{28}$. Thus, every element in $\Theta_{7}$ can be represented by some $\Sigma_{n}^{7}$ such that $E_{n}^{10}$ admits a cohomogeneity one metric with $K \geq 0$. However, this does not mean that $\Sigma_{n}^{7}$ admits a metric with $K \geq 0$ since the Gromoll-Meyer action $E_{n}^{10}$ is not isometric with respect to the Grove-Ziller metric. 


\section{INVARIANT SUBMANIFOLDS AND PARITY}

In this section we will see that the even/odd grading of the generalized GromollMeyer spheres $\Sigma_{n}^{7}$ is based on an elementary property of the maps $\rho_{n}$.

Consider the subsets

$$
\begin{aligned}
& E_{n}^{9}:=\left\{(u, v) \in E_{n}^{10} \mid u \in \operatorname{Im} \mathbb{H} \times \mathbb{H}\right\}, \\
& E_{n}^{8}:=\left\{(u, v) \in E_{n}^{10} \mid u \in \operatorname{Im} \mathbb{H} \times \operatorname{Im} \mathbb{H}\right\}
\end{aligned}
$$

of $E_{n}^{10} \subset \mathbb{S}^{7} \times \mathbb{S}^{7}$. These are the preimages of the subspheres

$$
\begin{aligned}
& \mathbb{S}^{6}=\left\{\left.\left(\begin{array}{c}
p \\
w
\end{array}\right)|p \in \operatorname{Im} \mathbb{H}, w \in \mathbb{H},| p\right|^{2}+|w|^{2}=1\right\}, \\
& \mathbb{S}^{5}=\left\{\left.\left(\begin{array}{c}
p_{1} \\
p_{2}
\end{array}\right)\left|p_{1}, p_{2} \in \operatorname{Im} \mathbb{H},\right| p_{1}\right|^{2}+\left|p_{2}\right|^{2}=1\right\}
\end{aligned}
$$

of $\mathbb{S}^{7} \subset \mathbb{H} \times \mathbb{H}$ under the principal bundle projection $E_{n}^{10} \rightarrow \mathbb{S}^{7}$.

Lemma 3.1. $E_{n}^{9}$ and $E_{n}^{8}$ are submanifolds of $E_{n}^{10}$ diffeomorphic to $\mathbb{S}^{6} \times \mathbb{S}^{3}$ and $\mathbb{S}^{5} \times \mathbb{S}^{3}$, respectively .

Proof. $E_{n}^{9} \rightarrow \mathbb{S}^{6}$ is a proper subbundle of $E_{n}^{10} \rightarrow \mathbb{S}^{7}$ and hence trivial.

Lemma 3.2. $E_{n}^{9}$ and $E_{n}^{8}$ are invariant under the free $\star$-action of $\mathbb{S}^{3}$ and under the - action of $\mathbb{Z}_{2} \times \mathbb{Z}_{2} \times \mathbb{S}^{3}$. Hence, the $\star$-quotients $\Sigma_{n}^{6}$ and $\Sigma_{n}^{5}$ are submanifolds of $\Sigma_{n}^{7}$ with a natural $\bullet$-action of $\mathbb{Z}_{2} \times \mathbb{Z}_{2} \times \mathrm{SO}(3)$.

Proof. Straightforward.

Lemma 3.3. As submanifolds of $\mathbb{S}^{7} \times \mathbb{S}^{7}$ we have

$$
\begin{gathered}
\ldots=E_{-3}^{9}=E_{-1}^{9}=E_{1}^{9}=E_{3}^{9}=\ldots, \\
\ldots=E_{-4}^{9}=E_{-2}^{9}=E_{0}^{9}=E_{2}^{9}=E_{4}^{9}=\ldots
\end{gathered}
$$

and the same identities also hold for $E_{n}^{8} \subset E_{n}^{9}$ and for the quotients $\Sigma_{n}^{6}$ and $\Sigma_{n}^{5}$.

Proof. This is an immediate consequence of the two basic identities

$$
\rho_{2 m+1}\left(\left(\begin{array}{c}
p \\
w
\end{array}\right)\right)=(-1)^{m}\left(\begin{array}{c}
p \\
w
\end{array}\right) \quad \text { and } \quad \rho_{2 m}\left(\left(\begin{array}{c}
p \\
w
\end{array}\right)\right)=(-1)^{m}\left(\begin{array}{l}
1 \\
0
\end{array}\right)
$$

for $\left(\begin{array}{c}p \\ w\end{array}\right) \in \mathbb{S}^{6} \subset \operatorname{Im} \mathbb{H} \times \mathbb{H}$.

Corollary 3.4. If $n$ is odd, $\Sigma_{n}^{5}$ is equivariantly diffeomorphic to the Brieskorn sphere $W_{3}^{5}$ with its natural $\mathbb{Z}_{2} \times \mathbb{Z}_{2} \times \mathrm{SO}(3)$-action. If $n$ is even, $\Sigma_{n}^{5}$ is equivariantly diffeomorphic to the Euclidean sphere $\mathbb{S}^{5} \subset \mathbb{R}^{3} \times \mathbb{R}^{3}$ where $\mathrm{SO}(3)$-acts diagonally on both $\mathbb{R}^{3}$-factors and each $\mathbb{Z}_{2}$-factor acts on one of the $\mathbb{R}^{3}$-factors.

Proof. From Lemma 7.4 of $[\mathrm{DP}]$ we know that $\Sigma_{1}^{5}$ is $\mathbb{Z}_{2} \times \mathbb{Z}_{2} \times \mathrm{SO}(3)$-equivariantly diffeomorphic to $W_{3}^{5}$. For $\Sigma_{0}^{5}$ we observe that

$$
E_{0}^{8}=\left\{\left(\begin{array}{cc}
p_{1} & 0 \\
p_{2} & q
\end{array}\right) \mid p_{1}, p_{2} \in \operatorname{Im} \mathbb{H}, q \in \mathbb{S}^{3}\right\}
$$

The natural embedding $\mathbb{S}^{5} \rightarrow E_{0}^{8},\left(\begin{array}{l}p_{1} \\ p_{2}\end{array}\right) \mapsto\left(\begin{array}{ll}p_{1} & 0 \\ p_{2} & 1\end{array}\right)$ identifies the $\star$-quotient of $E_{0}^{8}$ with $\mathbb{S}^{5}$.

Lemma 3.5. The subsphere $\Sigma_{n}^{5}$ is minimal in $\Sigma_{n}^{6}$ and $\Sigma_{n}^{7}$ for all $\{ \pm \mathbb{1}\} \times \mathrm{SO}(3)$ invariant Riemannian metrics on $\Sigma_{n}^{7}$. 
Proof. Analogous to the proof of Corollary3.4 in [DP this follows from the fact that $\Sigma_{n}^{5}$ is the union of orbits whose isotropy groups contain elements of the form $(-\mathbb{1}, \pm q)$.

\section{The GeOdesic Join StRUCture of $\Sigma_{n}^{7}$}

We will now study the geometry of a one parameter family of Riemannian metrics on $E_{n}^{10}$ and $\Sigma_{n}^{7}$ and use the results to prove Theorem 1 , Theorem 2 and Theorem 4 . The one parameter family of metrics is defined in such a way that the constructions of $\left[\mathrm{Du}\right.$ and $[\mathrm{DP}]$ for $\Sigma_{\mathrm{GM}}^{7}$ can be extended to all $\Sigma_{n}^{7}$.

We equip the total space of the principal bundle $E_{n}^{10} \rightarrow \mathbb{S}^{7}$ with the Riemannian metric $\langle\cdot, \cdot\rangle_{\nu}$ with $\nu>0$ defined by the following properties:

- The $\mathbb{S}^{3}$-fibers have constant curvature $\frac{1}{\nu}$.

- The horizontal distribution is given by the pull-back of the horizontal distribution of $\operatorname{Sp}(2)$ via the map $\rho_{n}$, i.e., we pull-back the principal bundle connection of $\operatorname{Sp}(2)$.

- The metric $\langle\cdot, \cdot\rangle_{\nu}$ induces on $\mathbb{S}^{7}$ the metric with constant curvature 1 by Riemannian submersion.

Such metrics are called connection metrics or Kaluza-Klein metrics.

The $\mathbb{Z}_{2} \times \mathbb{Z}_{2} \times \mathbb{S}^{3} \times \mathbb{S}^{3} \times \mathbb{S}^{3}$-action given in (3) and (4) is isometric with respect to the metric $\langle\cdot, \cdot\rangle_{\nu}$. In particular, the Gromoll-Meyer action $\star$ is isometric and $\Sigma_{n}^{7}$ inherits a Riemannian metric by Riemannian submersion, which will again be denoted by $\langle\cdot, \cdot\rangle_{\nu}$. The $\bullet$-action of $\mathbb{Z}_{2} \times \mathbb{Z}_{2} \times \mathbb{S}^{3}$ on $E_{n}^{10}$ is also isometric. Since the $\bullet$-action commutes with the $\star$-action, it induces an effective isometric $\mathbb{Z}_{2} \times \mathbb{Z}_{2} \times \mathrm{SO}(3)$-action on $\left(\Sigma_{n}^{7},\langle\cdot, \cdot\rangle_{\nu}\right)$.

Lemma 4.1. The common fixed point set of $\mathrm{SO}(3)$ in $\Sigma_{n}^{7}$ is the circle

$$
\Sigma_{n}^{1}:=\left\{\pi_{\Sigma_{n}^{7}}\left(\left(\begin{array}{cc}
\cos t & -\sin n t \\
\sin t & \cos n t
\end{array}\right)\right) \mid t \in \mathbb{R}\right\} .
$$

Hence, for any $\mathrm{SO}(3)$-invariant Riemannian metric on $\Sigma_{n}^{7}$, this circle $\Sigma_{n}^{1}$ is a simple closed geodesic.

Proof. $\pi_{\Sigma_{n}^{7}}(u, v)$ is a fixed point of $\mathrm{SO}(3)$ if and only if for every $q \in \mathbb{S}^{3}$ there is a $q^{\prime} \in \mathbb{S}^{3}$ such that $\left(q^{\prime} u \bar{q}^{\prime}, q^{\prime} v \bar{q}\right)=(u, v)$. It is easy to see from the second column of this equation that all elements of $\mathbb{S}^{3}$ occur for $q^{\prime}$. Therefore, $u$ must have two real components.

Note that the $\mathbb{Z}_{2} \times \mathbb{Z}_{2}$-action on $\Sigma_{n}^{1}$ is equivalent to the standard $\mathbb{Z}_{2} \times \mathbb{Z}_{2}$-action on $\mathbb{S}^{1}$. In particular, for each point $p \in \Sigma_{n}^{1}$ there is a natural antipode $-p$.

Theorem 4.2. Every unit speed geodesic $\gamma$ in $\left(\Sigma_{n}^{7},\langle\cdot, \cdot\rangle_{\nu}\right)$ with $\gamma(0)=p \in \Sigma_{n}^{1}$ is length minimizing on $[0, \pi[$ and we have $\gamma(\pi)=-p$ and $\gamma(2 \pi)=p$.

Proof. The proof is similar to the proofs of Theorem I in [Du and Theorem 2.1 in [DP. We lift $\gamma$ horizontally to a geodesic $\tilde{\gamma}$ in $E_{n}^{10}$ with

$$
\tilde{\gamma}(0)=\alpha(t):=\left(\begin{array}{cc}
\cos t-\sin n t \\
\sin t & \cos n t
\end{array}\right) \in E_{n}^{1} .
$$


That $\tilde{\gamma}$ is horizontal with respect to $E_{n}^{10} \rightarrow \Sigma_{n}^{7}$ means that the geodesic $\tilde{\gamma}$ passes perpendicularly through all $\star$-orbits. It is straightforward to check that

$$
\mathbb{S}^{3} \star \alpha(t)=\mathbb{S}^{3} \bullet \alpha(t)
$$

Thus, $\tilde{\gamma}$ passes perpendicularly through $\mathbb{S}^{3} \bullet \tilde{\gamma}(0)$. A geodesic that passes perpendicularly through one orbit passes perpendicularly through all orbits. Hence, $\tilde{\gamma}$ passes perpendicularly through all $\mathbb{S}^{3}$-orbits of the $\bullet$-action. In other words, $\tilde{\gamma}$ is horizontal to the principal fibration $E_{n}^{10} \rightarrow \mathbb{S}^{7}$. Hence, $\tilde{\gamma}$ projects to a geodesic $\beta$ in $\mathbb{S}^{7}$. By definition of $\langle\cdot, \cdot\rangle_{\nu}$ the sphere $\mathbb{S}^{7}$ inherits the metric with constant curvature 1 from $E_{n}^{10}$ by Riemannian submersion. Since all unit speed geodesics of $\mathbb{S}^{7}$ that start at $\beta(0)=\pi_{\mathbb{S}^{7}}(\alpha(t))$ pass through $\beta(\pi)=-\beta(0)$ at time $\pi$ we have $\beta(\pi)=\pi_{\mathbb{S}^{7}}(\alpha(t+\pi))$. Thus, $\tilde{\gamma}(\pi)$ is contained in $\mathbb{S}^{3} \bullet \alpha(t+\pi)=\mathbb{S}^{3} \star \alpha(t+\pi)$ and $\tilde{\gamma}(2 \pi)$ is contained in $\mathbb{S}^{3} \star \alpha(t+2 \pi)=\mathbb{S}^{3} \bullet \tilde{\gamma}(0)$. This shows $\gamma(\pi)=-\gamma(0)$ and $\gamma(2 \pi)=\gamma(0)$. Now let $\gamma$ be a unit speed geodesic in $\Sigma_{n}^{7}$ with $\gamma(0)=p$ and $\gamma_{1}(l)=-p$. By the construction above $\beta$ is a unit speed geodesic in $\mathbb{S}^{7}$ with $\beta(l)=-\beta(0)$. Hence, $l$ cannot be less than $\pi$.

Recall that the join $X * Y$ of two spaces $X$ and $Y$ is the quotient of $X \times Y \times[0,1] / \sim$ where $(x, y, 0) \sim\left(x, y^{\prime}, 0\right)$ and $(x, y, 1) \sim\left(x^{\prime}, y, 1\right)$ for all $x \in X$ and all $y \in Y$. For our purposes it is convenient to substitute $[0,1]$ by $\left[0, \frac{\pi}{2}\right]$.

Corollary 4.3. $\Sigma_{n}^{1}$ and $\Sigma_{n}^{5}$ have constant distance $\frac{\pi}{2}$. Moreover, the map $\Sigma_{n}^{1} * \Sigma_{n}^{5} \rightarrow$ $\Sigma_{n}^{7}$ that maps $(x, y, t)$ to $\gamma(t)$, where $\gamma:\left[0, \frac{\pi}{2}\right] \rightarrow \Sigma^{7}$ is the unique unit speed geodesic segment from $x$ to $y$, is an equivariant homeomorphism.

Proof. This follows from the construction in the proof of Theorem 4.2 if one recalls that the submanifolds $E_{n}^{1}$ and $E_{n}^{9}$ of $E_{n}^{10}$ project to the submanifolds

$$
\begin{aligned}
& \mathbb{S}^{1}=\left\{\left(\begin{array}{c}
\cos t \\
\sin t
\end{array}\right) \mid t \in \mathbb{R}\right\}, \\
& \mathbb{S}^{5}=\left\{\left.\left(\begin{array}{c}
p_{1} \\
p_{2}
\end{array}\right)\left|p_{1}, p_{2} \in \operatorname{Im} \mathbb{H},\right| p_{1}\right|^{2}+\left|p_{2}\right|^{2}=1\right\}
\end{aligned}
$$

of $\mathbb{S}^{7} \subset \mathbb{H}^{2}$ under the principal fibration $E_{n}^{10} \rightarrow \mathbb{S}^{7}$ and to the submanifolds $\Sigma_{n}^{1}$ and $\Sigma_{n}^{5}$ of $\Sigma_{n}^{7}$ under the principal fibration $E_{n}^{10} \rightarrow \Sigma_{n}^{7}$.

Theorem 4.2 and Corollary 4.3 together yield Theorem 4 from the introduction.

Corollary 4.4. $\Sigma_{n}^{7}$ is $\mathbb{Z}_{2} \times \mathbb{Z}_{2} \times \mathrm{SO}(3)$-equivariantly homeomorphic to $\mathbb{S}^{1} * \mathbb{S}^{5}$ if $n$ is even and to $\mathbb{S}^{1} * W_{3}^{5}$ if $n$ is odd. Here, the $\mathbb{Z}_{2} \times \mathbb{Z}_{2}$ acts on $\mathbb{S}^{1}$ in the standard way.

Proof. This is evident from Corollary 3.4 and Corollary 4.3

In particular, all $\Sigma_{n}^{7}$ with even $n$ are mutually equivariantly homeomorphic and that all $\Sigma_{n}^{7}$ with odd $n$ are mutually equivariantly homeomorphic. This proves Theorem 2 from the introduction.

Proof of Theorem 1. Consider the unit speed geodesic

$$
\beta(t)=\left(\begin{array}{c}
\cos t+p \sin t \\
w \sin t
\end{array}\right)
$$


in $\mathbb{S}^{7} \subset \mathbb{H}^{2}$ that emanates from the north pole with initial velocity $\left(\begin{array}{c}p \\ w\end{array}\right) \in \mathbb{S}^{6} \subset$ $\operatorname{Im} \mathbb{H} \times \mathbb{H}$. A lift $\tilde{\gamma}_{n}$ of this curve to $E_{n}^{10}$ with $\tilde{\gamma}_{n}(0)=\left(\begin{array}{ll}1 & 0 \\ 0 & 1\end{array}\right)$ is given by

$$
\tilde{\gamma}_{n}(t)=\left(\begin{array}{cc}
\cos t+p \sin t & -e^{n t p} \bar{w} \sin (n t) \\
w \sin t & \left.\frac{w}{|w|} e^{n t p}(\cos (n t)-p \sin (n t))\right) \frac{\bar{w}}{|w|}
\end{array}\right) .
$$

Here $e^{p}=\cos |p|+\frac{p}{|p|} \sin |p|$ denotes the exponential map of $\mathbb{S}^{3} \subset \mathbb{H}$ at 1 . Note that for $w=0$ equation (5) simply becomes $\tilde{\gamma}_{n}(t)=\left(\begin{array}{cc}e^{n t p} & 0 \\ 0 & 1\end{array}\right)$. Using the identity

$$
\tilde{\rho}_{n}\left(\tilde{\gamma}_{n}(t)\right)=\tilde{\gamma}_{1}(n t)
$$

for the map $\tilde{\rho}_{n}: E_{n}^{10} \rightarrow \operatorname{Sp}(2)$ defined in section 2 it is straightforward to verify that $\tilde{\gamma}_{n}$ is the unique horizontal lift of $\beta$ to $E_{n}^{10}$ with $\tilde{\gamma}_{n}(0)=\mathbb{1}$. Since the fibers of $E_{n}^{10} \rightarrow \mathbb{S}^{7}$ and $E_{n}^{10} \rightarrow \Sigma_{n}^{7}$ through $\tilde{\gamma}_{n}(0)=\left(\begin{array}{ll}1 & 0 \\ 0 & 1\end{array}\right)$ are the same (as sets), the geodesic $\tilde{\gamma}_{n}$ is horizontal with respect to both these fibrations. This shows that $\gamma_{n}=\pi_{\Sigma_{n}^{7}} \circ \tilde{\gamma}_{1}$ is a geodesic in $\Sigma_{n}^{7}$. Now, considering all possible unit initial vectors $\left(\begin{array}{c}p \\ w\end{array}\right) \in \mathbb{S}^{6} \subset$ $\operatorname{Im} \mathbb{H} \times \mathbb{H}$ and times $t \in\left[0, \frac{\pi}{2}\right]$ the geodesics $\gamma_{n}$ provide an embedding of a disk $D^{7}\left(\frac{\pi}{2}\right)$ into $\Sigma_{n}^{7}$ by Theorem 4.2 In the same way, the geodesics $\pi_{\Sigma^{7}} \circ\left(-\tilde{\gamma}_{n}\right) \circ(-\mathrm{id})$ provide another embedding of the same disk. By Theorem $4.2 \Sigma_{n}^{7}$ is the twisted sphere obtained by gluing these two embedded disks along their common boundary. One easily checks that

$$
\tilde{\gamma}_{n}\left(p, w, \frac{\pi}{2}\right)=q \star\left(-\tilde{\gamma}_{n}\left(-p^{\prime},-w^{\prime}, \frac{\pi}{2}\right)\right)
$$

for some $q \in \mathbb{S}^{3}$ if and only if $\left(p^{\prime}, w^{\prime}\right)=\sigma^{n}(p, w)$ where $\sigma$ is the exotic diffeomorphism of $\mathbb{S}^{6} \subset \operatorname{Im} \mathbb{H} \times \mathbb{H}$ first described in $[\mathrm{Du}$. This diffeomorphism $\sigma$ generates $\pi_{0}\left(\operatorname{Diff}_{+}\left(\mathbb{S}^{6}\right)\right)$. It is given by the formula

$$
\sigma(p, w):=\overline{\mathrm{b}(p, w)}(p, w) \mathrm{b}(p, w)
$$

where $\mathrm{b}(p, w)=\frac{w}{|w|} e^{\pi p} \frac{\bar{w}}{|w|}$ is an analytic formula for a generator of $\pi_{6}\left(\mathbb{S}^{3}\right)$. Hence, we have obtained $\Sigma_{n}^{7}$ by gluing two 7-disks with the $n$-th power of a generator of $\pi_{0}\left(\operatorname{Diff}_{+}\left(\mathbb{S}^{6}\right)\right) \approx \Theta_{7} \approx \mathbb{Z}_{28}$.

Remark 4.5. Let $G$ be a compact group acting smoothly on $\Sigma_{n}^{7}$ with $\mathbb{Z}_{2} \times \mathbb{Z}_{2} \times$ $\mathrm{SO}(3) \subset G$. Precisely as in DP, Lemma 3.7, one can show that $G$ leaves $\Sigma_{n}^{1}$ and $\Sigma_{n}^{5}$ invariant. Let $n \notin\{-1,0,1\}$. Comparing for different $p \in \Sigma_{n}^{1}$ the closing behaviour of geodesics that start at $p$ perpendicularly to $\Sigma_{n}^{1}$, one can see that $\mathbb{Z}_{2} \times \mathbb{Z}_{2}$ is the maximal compact group that acts isometrically on $\left(\Sigma_{n}^{7},\langle\cdot, \cdot\rangle_{\nu}\right)$ and effectively on the circle $\Sigma_{n}^{1}$. This difference from the cases $n=-1,0,1$ suggests that $\mathbb{Z}_{2} \times \mathbb{Z}_{2} \times \mathrm{SO}(3)$ is the full isometry group of $\left(\Sigma_{n}^{7},\langle\cdot, \cdot\rangle_{\nu}\right)$.

Remark 4.6. If we pull back the metric $\langle\cdot, \cdot\rangle_{\nu}$ on $\operatorname{Sp}(2)$ by the map $\tilde{\rho}_{n}$ then we obtain a degenerate metric $\langle\cdot, \cdot\rangle_{\nu}^{\prime}$ on $E_{n}^{10}$ that has the same geodesics through the circle $\Sigma_{n}^{1}$ as the metric $\langle\cdot, \cdot\rangle_{\nu}$. For $n \notin\{-1,0,1\}$ the metric $\langle\cdot, \cdot\rangle_{\nu}^{\prime}$ is degenerate precisely over $|n|-1$ subspheres in $\mathbb{S}^{7}$ whose first quaternionic components have constant real part. With such a metric $\Sigma_{n}^{7}$ looks like $n$ copies of $\Sigma_{\mathrm{GM}}^{7}$ stacked one on top of the other, i.e., like a degenerate connected sum of $n$ copies of $\Sigma_{\mathrm{GM}}^{7}$. 
Remark 4.7. The manifolds $\left(E_{n}^{9},\langle\cdot, \cdot\rangle_{\nu}\right)$ with even $n$ are not just mutually equal as submanifolds of $\mathbb{S}^{7} \times \mathbb{S}^{7}$ but also mutually equal as Riemannian manifolds. Hence, also the manifolds $\left(\Sigma_{n}^{6},\langle\cdot, \cdot\rangle_{\nu}\right)$ with even $n$ are all mutually equal as Riemannian manifolds. The analogous statements hold for odd $n$.

\section{Comparison to the exotic Milnor and Brieskorn 7-spheres}

In this section we compare the equivariant topology of the spheres $\Sigma_{n}^{7}$ with the equivariant topology of the Milnor spheres $M_{d}^{7}$ and the Brieskorn spheres $W_{6 n-1,3}^{7}$ and prove Theorem 5 and Theorem 6 of the introduction.

Recall from the introduction that the Milnor spheres $M_{d}^{7}$ admit natural $\{ \pm \mathbb{1}\} \times$ $\mathrm{SO}(3)$-actions. Davis $\mathrm{Da}$, has shown that these actions can be extendend to $\mathrm{GL}(2, \mathbb{R}) \times \mathrm{SO}(3)$-actions. In the first chart the $\mathrm{GL}(2, \mathbb{R})$-action is given by

$$
\left(\begin{array}{ll}
a & c \\
b & d
\end{array}\right) \bullet(u, v)=\left(\frac{a u+c}{b u+d}, \operatorname{det}\left(\begin{array}{ll}
a & c \\
b & d
\end{array}\right)\left(\frac{b u+d}{|b u+d|}\right)^{k} v\left(\frac{b u+d}{|b u+d|}\right)^{l}\right)
$$

and in the second one by

$$
\left(\begin{array}{ll}
a & c \\
b & d
\end{array}\right) \bullet(u, v)=\left(\frac{b+d u}{a+c u}, \operatorname{det}\left(\begin{array}{ll}
a & c \\
b & d
\end{array}\right)\left(\frac{a+c \bar{u}}{|a+c \bar{u}|}\right)^{k} v\left(\frac{a+c \bar{u}}{|a+c \bar{u}|}\right)^{l}\right) .
$$

Note that our definition of the action differs from the definition given by Davis by the factor $\operatorname{det}\left(\begin{array}{ll}a & c \\ b & d\end{array}\right)$. The reason is that with our definition the identification between $M_{3}^{7}$ and the Gromoll-Meyer sphere $\Sigma_{\text {GM }}^{7}$ given in [GM] becomes an $\mathrm{O}(2) \times \mathrm{SO}(3)$ equivariant diffeomorphism while without the determiant factor the identification is only $\mathrm{SO}(2) \times \mathrm{SO}(3)$-equivariant. Moreover, note that the map $M_{d}^{7} \rightarrow M_{-d}^{7}$ given by $(u, v) \mapsto(\bar{u}, \bar{v})$ in both charts is an $\mathrm{GL}(2, \mathbb{R}) \times \mathrm{SO}(3)$-equivariant diffeomorphism.

Theorem 5.1. In every Milnor sphere $M_{d}^{7}$ there is a unique invariant submanifold $M_{d}^{5}$ which is $\mathrm{O}(2) \times \mathrm{SO}(3)$-equivariantly diffeomorphic to the Brieskorn sphere $W_{|d|}^{5}$ with the $\mathrm{O}(2) \times \mathrm{SO}(3)$-action given in (1)). This submanifold $M_{d}^{5}$ is minimal for any $\{ \pm \mathbb{1}\} \times \mathrm{SO}(3)$-invariant Riemannian metric on $M_{d}^{7}$.

Proof. It suffices to consider the case $d>0$. Let $M_{d}^{5}$ be the submanifold of $M_{d}^{7}$ given by the equations $\operatorname{Re} v=0$ and $\operatorname{Re} u v=0 \mathrm{n}$ both charts (it is essential here that $k+l=1)$. Hirsch and Milnor HMi proved that $M_{d}^{5}$ is homeomorphic and hence (because exotic spheres do not exist in dimension 5 ) diffeomorphic to $\mathbb{S}^{5}$. It is straightforward to check that $M_{d}^{5}$ is invariant under the $\mathrm{SO}(2) \times \mathrm{SO}(3)$-action. Consider the curve $\alpha$ in $M_{d}^{5}$ which is given by $\alpha(s)=(i \tan s, j)$ in the first chart. The isotropy groups along $\alpha$ are

$$
K_{-}=\left\{\left(\mathbb{1}, \pm e^{j \tau}\right)\right\} \cup\left\{\left(-\mathbb{1}, \pm i e^{j \tau}\right)\right\} \cup\left\{\left(\left[\begin{array}{rr}
1 & 0 \\
0 & -1
\end{array}\right], \pm e^{j \tau}\right)\right\} \cup\left\{\left(\left[\begin{array}{rr}
-1 & 0 \\
0 & 1
\end{array}\right], \pm i e^{j \tau}\right)\right\}
$$

at $s=0$,

$$
H=\left\{(\mathbb{1}, \pm 1),(-\mathbb{1}, \pm i),\left(\left[\begin{array}{rr}
1 & 0 \\
0 & -1
\end{array}\right], \pm j\right),\left(\left[\begin{array}{rr}
-1 & 0 \\
0 & 1
\end{array}\right], \pm k\right)\right\}
$$

for $0<s<\frac{\pi}{4}$, and

$$
K_{+}=\left\{\left(\left[\begin{array}{cr}
\cos \theta & -\sin \theta \\
\sin \theta & \cos \theta
\end{array}\right], \pm e^{-\frac{d}{2} i \theta}\right)\right\} \cup\left\{\left(\left[\begin{array}{cr}
\cos \theta & -\sin \theta \\
\sin \theta & \cos \theta
\end{array}\right] \cdot\left[\begin{array}{rr}
1 & 0 \\
0 & -1
\end{array}\right], \pm e^{-\frac{d}{2} i \theta} j\right)\right\}
$$


at $s=\frac{\pi}{4}$. Now consider the Brieskorn sphere $W_{d}^{5}$ with the $\mathrm{O}(2) \times \mathrm{SO}(3)$-action given in (11) and the curve

$$
\beta(s)=\left(s, 0, \frac{1}{\sqrt{2}} \sqrt{1-s^{2}-s^{d}},-\frac{i}{\sqrt{2}} \sqrt{1-s^{2}+s^{d}}\right)
$$

on the interval $\left[s_{-}, 0\right]$ where $s_{-}<0$ is the root of $1-s^{2}+s^{d}$. Straightforward computations show that the isotropy groups along $\beta$ are the same as the isotropy groups along $\alpha$. This proves that $M_{d}^{5}$ and $W_{d}^{5}$ are equivariantly diffeomorphic. The uniqueness and minimality of $M_{d}^{5}$ follows from the following fact: The fixed point set of any element of the form $(-\mathbb{1}, \pm q)$ is contained in $M_{d}^{5}$ and even more $M_{d}^{5}$ can be seen to be the union of orbits whose isotropy groups contains such elements.

Proof of Theorem 5. The involution $(-11, \pm i)$ is contained in $M_{d}^{5} \approx W_{|d|}^{5}$. The fixed point set of $(-\mathbb{1}, \pm i)=\left(-\mathbb{1}, \operatorname{diag}(1,-1,-1)\right.$ in $W_{|d|}^{5}$ is the $W_{|d|}^{3}$ given by the equation $z_{1}=0$ and hence diffeomorphic to a lens space with fundamental group $\mathbb{Z}_{|d|}$.

The Milnor sphere $M_{d}^{7}$ have direct analogues $M_{d}^{15}$ in dimension 15. They are obtained by gluing two copies of $\mathbb{O} \times \mathbb{S}^{7}$ along $(\mathbb{O} \backslash\{0\}) \times \mathbb{S}^{7}$ by the map (2). Precisely as above each $M_{d}^{15}$ admits a smooth action of $\mathrm{O}(2) \times \mathrm{G}_{2}$ (see $\mathrm{Da}$ ).

Theorem 5.2. In every $M_{d}^{15}$ there is a unique invariant submanifold $M_{d}^{13}$ which is $\mathrm{O}(2) \times \mathrm{G}_{2}$-equivariantly diffeomorphic to the Brieskorn sphere $W_{|d|}^{13}$ with the action of $\mathrm{O}(2) \times \mathrm{G}_{2} \subset \mathrm{O}(2) \times \mathrm{SO}(7)$ given analogously to (1). This submanifold $M_{d}^{13}$ is minimal for any $\{ \pm \mathbb{1}\} \times \mathrm{G}_{2}$-invariant Riemannian metric on $M_{d}^{15}$.

Proof. Analogous to the proof of Theorem 5.1

Finally, we turn to the Brieskorn spheres $W_{6 n-1,3}^{7}$ and prove Theorem 6 from the introduction.

Proof of Theorem 6. The involution $\left(\begin{array}{rrr}1 & 0 & 0 \\ 0 & -1 & 0 \\ 0 & 0 & -1\end{array}\right) \in \mathrm{SO}(3)$ on $W_{6 n-1,3}^{7}$ is given by

$$
\left(w, z_{0}, z_{1}, z_{2}, z_{3}\right) \mapsto\left(w, z_{0}, z_{1},-z_{2},-z_{3}\right) .
$$

Its fixed point set is thus identical to $W_{6 n-1,3,2}^{3}$, which is the intersection of the unit sphere $\mathbb{S}^{5}$ in $\mathbb{C}^{3}$ with the complex hypersurface

$$
w^{6 n-1}+z_{0}^{3}+z_{1}^{2}=0 .
$$

Milnor Mi2 has shown that $W_{5,3,2}^{3}$ is diffeomorphic to Poincare dodecahedral space and that the universal covering space of $W_{6 n-1,3,2}^{3}$ is non-compact if $n>1$.

\section{REFERENCES}

[Bs] A. L. Besse, Manifolds all of whose geodesics are closed, Ergebnisse der Mathematik und ihrer Grenzgebiete 93, Springer, Berlin-New York, 1978.

[Bk] E. Brieskorn, Beispiele zur Differentialgeometrie von Singularitäten, Invent. Math. 2 (1966), 1-14.

[Da] M. W. Davis, Some group actions on homotopy spheres of dimension seven and fifteen, Am. J. Math. 104 (1982), 59-90. 
[Du] C. E. Durán, Pointed Wiedersehen metrics on exotic spheres and diffeomorphisms of $\mathbb{S}^{6}$, Geom. Dedicata 88 (2001), 199-210.

[DMR] C. E. Durán, A. Mendoza, A. Rigas, Blakers-Massey elements and exotic diffeomorphisms of $S^{6}$ and $S^{14}$ via geodesics, Trans. Amer. Math. Soc. 356 (2004), 5025-5043.

[DP] C. E. Durán, T. Püttmann, A minimal Brieskorn 5-sphere in the Gromoll-Meyer sphere and its applications, arXiv:math.DG/0606769

[EK] J. Eells, N. H. Kuiper, An invariant for certain smooth manifolds, Ann. Mat. Pura Appl. 60 (1962), 93-110.

[GM] D. Gromoll, W. Meyer, An exotic sphere with nonnegative curvature, Ann. Math. 100 (1974), 401-406.

[GZ] K. Grove, W. Ziller, Curvature and symmetry of Milnor spheres, Ann. of Math. 152 (2000), 331-367.

[HMi] M. Hirsch, J. Milnor, Some curious involutions of spheres, Bull. Amer. Math. Soc. 70 (1964), 372-377.

[HMa] F. Hirzebruch, K. H. Mayer, O(n)-Mannigfaltigkeiten, exotische Sphären und Singularitäten, Lecture Notes in Mathematics 57, Springer, Berlin 1968.

$[\mathrm{Hu}]$ S. T. Hu, Homotopy theory, Pure and Applied Mathematics VIII, Academic Press, New York, 1959.

[KZ] V. Kapovitch, W. Ziller, Biquotients with singly generated rational cohomology, Geom. Dedicata 104 (2004), 149-160.

[KM] M. A. Kervaire, J. W. Milnor, Groups of homotopy spheres I, Ann. of Math. 77 (1963), 504-537.

[Mi1] J.W. Milnor, On manifolds homeomorphic to the 7-sphere, Ann. of Math. 64 (1956), 399-405.

[Mi2] J. W. Milnor, On the 3-dimensional Brieskorn manifolds $M(p, q, r)$. Knots, Groups and 3-Manifolds (L. P. Neuwirth, ed.), Annals of Mathematical Studies 84, 175-225. Princeton University Press, Princeton, NJ, 1975.

[Ri] A. Rigas, $S^{3}$-bundles and exotic actions, Bull. Soc. Math. France 112 (1984), 69-92; correction by T. E. Barros, Bull. Soc. Math. France 129 (2001), 543-545.

[St] E. Straume, Compact differentiable transformation groups on exotic spheres, Math. Ann. 299 (1994), 355-389.

[To] B. Totaro, Cheeger manifolds and the classification of biquotients, J. Differential Geom. 61 (2002), 397-451.

[Wa] C. T. C. Wall, Surgery on compact manifolds, Mathematical surveys and monographs 69, AMS, Providence, 1999

imeCC-Unicamp, Praça Sergio Buarque de Holanda, 651, Cidade Universitária Barão Geraldo, Caixa Postal: 6065 13083-859 Campinas, SP, Brasil

E-mail address: cduran@ime.unicamp.br

Mathematisches Institut, Universität Bonn, D-53115 Bonn, Germany

E-mail address: puttmann@math.uni-bonn.de

imeCC-Unicamp, Praça Sergio Buarque de Holanda, 651, Cidade Universitária Barão Geraldo, Caixa Postal: 6065 13083-859 Campinas, SP, Brasil

E-mail address: rigas@ime.unicamp.br 